\title{
Asparaginase-induced pancreatitis in children: Surgical perspective and review of literature
}

\author{
Dayang A. Aziz ${ }^{1}$, Ian Chik ${ }^{1}$, Faizah MZ², CKhai Loh ${ }^{3}$, Swee Fong Tang ${ }^{3}$ and AL Zarina \\ ${ }^{1}$ Department of Surgery, Universiti Kebangsaan Malaysia Medical Centre, Malaysia \\ ${ }^{2}$ Department of Radiology, Universiti Kebangsaan Malaysia Medical Centre, Malaysia \\ ${ }^{3}$ Department of Paediatrics, Universiti Kebangsaan Malaysia Medical Centre, Malaysia
}

\begin{abstract}
Asparaginase is an important chemotherapeutic agent used in the treatment of haematological malignancies such as acute lymphoblastic leukemia. As with many cytotoxic medications, asparaginase has its adverse effects. Pancreatitis is one of the complications; often difficult to diagnose, leading to morbidity and possible mortality. In this case series, six paediatric patients with acute leukaemia are presented and management issues in relation to diagnostic challenge and surgical intervention for asparaginase-associated pancreatitis are discussed. The first three patients were managed differently from the last three. Low threshold for suspicion of pancreatitis is required, prompt cessation of asparaginase, early CT imaging of pancreas and serial level of CRP have shown to improve diagnosis and helped plan management of patients.
\end{abstract}

\section{Introduction}

Asparaginase is an enzyme that has been used in treatment regimens for childhood acute lymphoblastic leukemia (ALL) since 1961 [1]. It is usually used in combination with daunorubicin, vincristine (VCR) and dexamethasone and is also used in the treatment of lymphoma. The enzyme is derived from bacteria and derivations from either Escherichia coli (E.coli) or Erwinia chrysanthemi is used.

The use of this enzyme has improved remission rates ranging from $83 \%$ to $95 \%$ of childhood ALL patients [2]. However, asparaginase is not without complications. The use of asparaginase may cause a spectrum of allergic reactions in about 20 to $40 \%$ of patients, which includes erythema at site of injection, urticaria, bronchospasm and even anaphylaxis [3-6]. Other reported complications include haemostatic abnormality, hyperglycaemia, abnormal lipid metabolism and pancreatitis.

In this case series, the importance of early diagnosis and management of pancreatitis will be discussed in detail. It is important to be vigilant of pancreatitis as a complication as it is difficult to diagnose, and has morbid sequelae if not treated immediately. Once diagnosed, cessation of asparaginase with management of pancreatitis may prevent complications from pancreatitis.

\section{Case 1}

A 6-year-old boy was diagnosed to have late relapse of ALL whilst on maintenance phase of chemotherapy, based on the UKALL 2003 Regimen B. He was subsequently started on the R3 protocol of induction chemotherapy, which comprised intravenous idarubicin, oral dexamethasone $\left(20 \mathrm{mg} / \mathrm{m}^{2} /\right.$ day in divided doses, given for a total of 28 days), intrathecal (IT) methotrexate (MTX), intravenous vincristine sulfate and intramuscular (IM) asparaginase. The latter $\left(6000 \mathrm{U} / \mathrm{m}^{2} / \mathrm{d}\right)$ was given on an alternate day basis from day 3 till 25 of induction amounting to a total of 12 doses.
The patient had received a total of eight doses of asparaginase, when he complained of worsening abdominal pain. The pain was in the umbilical region, radiated to the back and was associated with a decrease in appetite. He had been complaining of similar but milder pain for a week, which worsened with accumulative doses of asparaginase. He was treated as having steroid induced gastritis as the initial serum amylase level was normal and there was alleviation of pain with proton pump inhibitors (PPI).

Clinically, he was afebrile and haemodynamically stable. Physical examination revealed epigastric tenderness but no guarding. There was no ascites, no Cullen nor Grey Turner sign. Investigations revealed that the serum amylase was raised (846 U/L) and a diagnosis of asparaginase- induced pancreatitis (AIP) was made. His other blood investigations showed: low serum albumin $30 \mathrm{~g} / \mathrm{L}$; glucose level 9 $\mathrm{mmol} / \mathrm{L}$; lactate dehydrogenase $263 \mathrm{U} / \mathrm{L}$ and neutropaenic (ANC 0.1 $\left.\mathrm{x} 10^{\wedge} 9 / \mathrm{L}\right)$. His Glasgow Imrie score was one that of mild pancreatitis (scores for hypoalbuminaemia).

Abdominal ultrasound revealed peripancreatic fluid, suggestive of acute pancreatitis. He was kept nil by mouth and morphine infusion was administered for analgesia. Surgical referral was made at this point. Computerised tomographic scan (CT) of the abdomen was planned in view of clinical suspicion of necrotising pancreatitis as patient deteriorated rapidly, developing hypotension that was not responsive to fluid challenge and requiring inotropic support. Unfortunately the

Correspondence to: Dayang Anita Abdul Aziz, Paediatric Surgery Unit, Department of Surgery, Universiti Kebangsaan Malaysia Medical Centre (UKMMC), Jalan Yaacob Latiff, Bandar Tun Razak, Cheras, Kuala Lumpur 56000 Malaysia, Tel: +6012-305-9439; E-mail: dayanganita@yahoo.co.uk

Key words: asparaginase, pancreatitis, diagnosis, surgery

Received: July 07, 2016; Accepted: August 02, 2016; Published: August 05, 2016 
patient's condition worsened quickly and required ventilatory support and developed coagulopathy, requiring transfusion of multiple blood products. He was unable to undergo interventional procedure (patient was planned for necrosectomy of pancreas following CT findings suggestive of necrotising pancreatitis) due to his clinical condition and finally succumbed to his illness.

\section{Case 2}

A 6-year-old boy with background history of glucose-6-phosphate dehydrogenase (G6PD) deficiency and T-cell lymphoblastic lymphoma stage 3 (diagnosed 6 months earlier), presented with persistent central abdominal pain, bilious vomiting, and constipation for 2 days duration. He was febrile, normotensive but tachycardic. Abdominal examination revealed distension with epigastric and periumbilical tenderness. His serum amylase was normal (30 U/L).

During induction phase, patient was given 30 days of prednisolone $\left(20 \mathrm{mg} / \mathrm{m}^{2} /\right.$ day in divided doses), in addition to cyclical doses of VCR, daunorubicin, IT MTX and 8 doses of IM asparaginase $(10,000$ $\mathrm{U} / \mathrm{m}^{2} /$ day). The patient had abdominal discomfort following each administration of asparaginase. A provisional diagnosis of AIP was made in view of elevated serum amylase (from 161 to $245 \mathrm{U} / \mathrm{L}$ ) although the ultrasound only revealed enlarged peripancreatic lymph nodes with specks of calcification and minimal pelvic free fluid. As his symptoms improved following empirical treatment with PPI, he was subsequently managed as having steroid-induced gastritis.

After patient had been started on new phase of treatment, patient persisted to have sudden abdominal pain with non-bilious vomitus, resulting in paediatric surgical team being consulted. Despite normal serum amylase $(25,30 \mathrm{U} / \mathrm{L})$ levels and normal corrected calcium levels $(2.38 \mathrm{mmol} / \mathrm{L})$, provisional diagnosis of AIP was made, and an ultrasound scan done at this point revealed minimal free fluid in the perisplenic and pelvic region.

In view of a persistent colicky abdominal pain over one month, which evolved to sudden onset of vomiting, a pancreatic CT scan was requested to investigate for pancreatic pseudocyst, a known sequelae of pancreatitis. His CT scan revealed abnormal looking pancreas with pancreatic pseudocyst in the lesser sac measuring $9 \times 5 \times 8 \mathrm{~cm}$ with multiple smaller cysts seen lateral, medial and inferior to the large cyst. There was no free fluid seen in the CT.

As the patient was symptomatic, he underwent marsupialisation of the pseudocyst. The pseudocyst ruptured spontaneously intraoperatively necessitating lavage of the peritoneal cavity. There were areas of necrosis in the body of pancreas requiring necrosectomy. Post operatively, his recovery was uneventful but he had early satiety during meals. A follow- up abdominal ultrasound revealed a new pseudocyst $(4.6 \times 1.1 \mathrm{~cm})$. Unfortunately his family declined further treatment of his lymphoma and had defaulted oncology and surgical follow-up.

\section{Case 3}

A 12-year-old girl, diagnosed with ALL was commenced on UKALL (2003) Regimen B protocol. She was treated with IT MTX, IVI cyclophosphamide IV VCR IV doxorubicin, oral 6-mercaptopurine and IM Asparaginase $\left(6000 \mathrm{U} / \mathrm{m}^{2} /\right.$ day $)$.

She had received a total of eleven doses of asparaginase, which was uneventful. She was then started on consolidation phase and on her second dose of asparaginase (After one month of rest), she developed severe epigastric pain and vomiting. On examination, she was febrile, normotensive but tachycardic. On palpation, her epigastrium was tender, mildly distended with ascites on percussion. She was diagnosed to have acute pancreatitis with a serum amylase of $303 \mathrm{U} / \mathrm{L}$, and urine amylase of $5284 \mathrm{U} / \mathrm{L}$. Ionised serum calcium was low $(0.68 \mathrm{mmol} / \mathrm{L})$ and she was hyperglycaemic (capillary blood glucose readings above 20 $\mathrm{mmol} / \mathrm{L}$ ). Abdominal ultrasound revealed right pleural effusion at base and ascites but no peripancreatic fluid was seen.

She was treated as AIP. IV calcium gluconate, insulin infusion and IV cefepime and amikacin were administered also administered on this patient apart from the standard pancreatitis conservative treatment.

The subsequent dose of asparaginase was given two weeks following resolution of acute pancreatitis. Unfortunately three months later, she complained of recurrent right hypochondrium pain over duration of two weeks which worsened following meals. Abdominal examination was unremarkable; based on her past history; a diagnosis of pancreatic pseudocyst was made. Serum amylase was $634 \mathrm{U} / \mathrm{L}$. There was no free fluid on abdominal ultrasound; unfortunately, the body and tail of pancreas was not well visualized. She was treated symptomatically but due to non-resolution of pain and elevated serum amylase (417 U/L) and urine amylase (515 U/L), abdominal CT was requested which revealed free fluid at peripancreatic region on the right side of lesser sac and ascites; there was no pseudocyst seen. During this period of time, two doses of IM asparaginase were administered.

Unfortunately she became febrile with worsening of abdominal pain. There was clinical evidence of ascites and blood investigations revealed: uncompensated metabolic acidosis, hypoalbuminaemia (24 $\mathrm{g} / \mathrm{L}$ ), serum amylase of $135 \mathrm{U} / \mathrm{L}$. INR 2.0, PT 21.3, APTT 38.9, while urine amylase was $416 \mathrm{U} / \mathrm{L}$ and neutropaenia (neutropaenia, TWC 0.5 $\times 10^{\wedge} 9 / \mathrm{L}$, ANC $\left.0.1 \times 10^{\wedge} 9 / \mathrm{L}\right)$. Ultrasound abdomen done showed no pseudocyst, but free fluid with peripancreatic collection.

The patient required inotropic support, fresh frozen plasma for coagulopathy and intravenous tazosin and amikacin. She was treated as acute on chronic pancreatitis secondary to asparaginase. Despite omission of asparaginase, she had recurrent severe abdominal pain, which was alleviated by leaning forward. Her serum amylase was 254 $\mathrm{U} / \mathrm{L}$ and abdominal ultrasound revealed pancreatic pseudocyst (3.9 $\times 1.7 \mathrm{~cm}$ ) which was then drained percutaneously. Serial abdominal ultrasound subsequently, showed no recurrence of pseudocyst. She had absolute omission of asparaginase throughout her chemotherapy and had no further recurrence of pancreatitis.

Subsequent to these three cases, we have then adopted an improvement to our surgical approach at managing possible AIP whereby level of C-reactive protein (CRP) and CT abdomen for assessment of pancreas were done early at the point of surgical referral. Our paediatric oncology colleagues also adopt early surgical referral. Case 4, 5 and 6 patients (all patients with ALL) went for immediate CT abdomen which revealed swollen pancreas with peripancreatic fluids. Level of serum amylase no longer was considered as an important marker. We also looked at serial level of CRP. These three patients were diagnosed as AIP when they have the triad of abdominal pain, elevated CRP and positive CT imaging of pancreas. They were kept nil by mouth for a few days, started on total parenteral nutrition and given intravenous analgesia (morphine). In patient Case 4 and Case 5, serial CRPs were raised around the levels of $18-20 \mathrm{mg} / \mathrm{dl}$ even when serum amylase was within normal limits at the point of diagnosis and the CRP levels normalised upon improvement of symptoms. In patient Case 6, serial CRPs were persistently high around $30 \mathrm{mg} / \mathrm{dl}$ even when patient's symptoms improved and this was attributed to presence of ongoing fungal infection in the patient's lung. 


\section{Discussion}

Asparaginase is an important chemotherapeutic agent in the treatment of both ALL and lymphoma. Pancreatitis is seen in about $5-18 \%$ of patients undergoing asparaginase treatment (both E.coli and Erwinase) [4,7-9] In a study by Kearney et al. [10] involving paediatric ALL patients enrolled into four different treatment protocols, pancreatitis can occur as early as after the first dose of asparaginase, mostly in the intensification period (with either E.coli or Erwinase $25000 \mathrm{IU} / \mathrm{m}^{2}$ per week - our centre uses leunase, which is E.coli, which has the same dose as Erwinase). It has been suggested that asparaginaseinduced pancreatitis may be due to an underlying predisposition rather than cumulative dose. It should be noted however, the dosage used differed from patients described in our case series. In this case series, 2 of our patients had received 8 doses prior to symptoms, while one had 11 doses with rest of 1 month period, before receiving 2 more doses and developing symptoms. Again, the patient's population quoted was in Boston, America, which would predominantly be a Caucasian population in comparison to our case series, which were Asians (specifically Malay, Chinese and Indian).

Although pancreatitis is a recognised complication of asparaginase, it is often missed as it is not readily confirmed by biochemical markers or imaging. The sequela of AIP includes haemorrhagic pancreatitis, pancreatic pseudocyst and necrotising pancreatitis. Haemorrhagic pancreatitis may be diagnosed by the appearance of Cullen or Grey Turner sign, which may lead to hemorrhagic shock if severe bleeding occurs. Whereas in a patient with pancreatic pseudocyst, often complain of anorexia with early satiety, and may have a palpable mass or fullness on palpation of the epigastrium. Early changes to the pancreas are best confirmed via an abdominal CT as illustrated in our patients. Necrotising pancreatitis is the most severe presentation of pancreatitis; it usually presents with recurrent bouts of pancreatitis in the first two weeks, followed by organ dysfunction and systemic complications. This was seen in our first case, where the patient had a rapid deterioration resulting in his demise.

As illustrated in our first three patients, pancreatitis was not the provisional diagnosis upon initial presentation; in fact the diagnosis only came into focus with the occurrence of complications. This posed a diagnostic dilemma amongst clinicians in the approach of a child with a haematological malignancy, who presents with epigastric or periumbilical pain. Paediatric patients with haematological malignancies often have concurrent administration of high dose steroids, which can lead to steroid induced gastritis. It is of utmost importance then, to have a high index of suspicion for pancreatitis as it is not unusual to have normal biochemical profile and imaging (ultrasound) results in the early stages.

Morimoto et al. [11] described two paediatric patients in whom serum amylase was normal and was only elevated eight to eleven days following initial presentation. In both their cases, the level of serum amylase did not reach the three times range of normal upper limit (2.2 to 2.3 times the normal upper limit) as per definition used by many clinicians. Similarly, serum lipase was also not raised until the seventh day of illness. Findings of normal serum amylase were also reported by Abhayakumar et al. [12]. Morimoto et al. [11] suggested the use of serum elastase as an additional biochemical marker to serum amylase and lipase, as it may detect sub-clinical or early pancreatitis caused by asparaginase as serum elastase levels were elevated before serum amylase or lipase. However, serum elastase has not been fully evaluated and is not available in many institutions. Serum lipase was also not readily available at our centre, hence was not used to aid in the diagnosis of our patients.

The Glasgow Imrie score can be used to score the severity of pancreatitis and calculates the chance of complications or mortality. The scoring system takes into account, age, total white count, arterial partial pressure of oxygen, serum calcium and albumin levels, blood glucose and urea levels, and lactate dehydrogenase. However, a previous calculation shows the sensitivity, specificity, positive and negative predictive value were $56 \%$, $98 \%$, $94 \%$ and $80 \%$ respectively (done in an adult population with alcoholic pancreatitis) [13]. It is important for prognostication, and should be done for every patient suspected of pancreatitis in the older age group of children. It should be noted that in the original Glasgow IMRIE scoring, age of patients in the sample were $52.3 \pm$ SD 18.6 years [14]. Serum amylase does not account for severity and hence, serial amylase is not usually performed. There has however, been correlation of severity of pancreatitis seen on CT scan with peak amylase level [10]. Findings include pancreatic enlargement, decreased echogenicity, loss of pancreatic contours, associated free fluid and pseudocyst formation.

$C$ reactive protein (CRP) has been studied previously as a diagnostic marker for pancreatitis. It is usually increased in inflammation, however noted to reach its peak at around 72 hours after onset of symptoms. In a paper by Fisic et al. [15] adult patients with acute pancreatitis caused by alcohol or biliary causes were studied for possible predictive biochemical markers. CRP was elevated in both mild and severe pancreatitis (reference value taken at $<0.5 \mathrm{mg} / \mathrm{dl}$ ), although statistically significant increase in concentration by third day is seen. From the same paper, the sensitivity and specificity of CRP reported is $54 \%$ and $84 \%$ respectively. In our patients, CRP was raised for all patients except for Case 3 in which the level was not done. Trend of CRP was not charted in the first three patients, but from the limited data, it can be noted that there was a raise in CRP, even when serum amylase was not raised. In the last three patients early level of CRP was a helpful indicator to assist diagnosis subsequent serial level of CRP seemed to correlate with clinical progress. This has helped us to plan treatment whether to surgically intervene or not (Table 1).

Imaging as a method for diagnosis of asparaginase-induced pancreatitis (AIP) has limited experience. In a paper by Kearney et al. [10], 39\% of patients ( 9 of 23 paediatric ALL) had normal ultrasound findings (pancreatitis diagnosed within first 10 weeks of consolidation phase), while both patients reported by Tsuyoshi Morimoto et al. [11] also had normal abdominal ultrasound and computed tomography (CT). Oh et al. [16], concluded that $87.5 \%$ of patients diagnosed to have AIP with concurrent CT scan had a positive CT result, which included peripancreatic fluids, oedematous pancreas, pancreatic parenchymal necrosis and infarct. As with the patients described in our case series, the diagnosis of pancreatitis was done via triad of clinical assessment, aided by abdominal CT (interstitial oedematous pancreatitis) and elevated CRP level. CT was also done for evaluation of complications of pancreatitis such as hemorrhagic pancreatitis, pseudocyst or necrotising pancreatitis.

\section{Conclusion}

It is important to recognise asparaginase-induced pancreatitis, as failure to diagnose maylead to significant morbidity and mortality. There should be cessation of asparaginase once a diagnosis of pancreatitis is made. Recommencement of asparaginase may however be considered on a case to case basis as previous studies have shown event free survival of the primary disease may be affected in those who did not 
Table 1. Correlation between patient's symptoms, CRP level and CT Imaging of the pancreas.

\begin{tabular}{|c|c|c|c|c|c|c|}
\hline & Dose (U/m $/ \mathbf{m}^{2}$ day) & Symptoms & Amylase (U/L) & CRP (mg/dl) & CT abdomen & Surgical treatment \\
\hline Patient 1 & 6000 & Central abdominal pain & $\begin{array}{l}150\left(6^{\text {th }}\right) \\
846\left(8^{\text {th }}\right)\end{array}$ & $5.2 \mathrm{mg} / \mathrm{dl}$ & Necrotising pancreatitis & Patient expired \\
\hline Patient 2 & 10000 & Central abdominal pain & $161-245\left(8^{\text {th }}\right)$ & $0.15-12.88$ & $\begin{array}{l}\text { Pancreatic pseudocyst with } \\
\text { necrotic body of pancreas }\end{array}$ & $\begin{array}{l}\text { Laparotomy, peritoneal washout } \\
\text { and necrosectomy }\end{array}$ \\
\hline Patient 3 & 6000 & $\begin{array}{l}\text { Severe epigastric pain and } \\
\text { vomiting }\end{array}$ & 303 & Not taken & Pancreatic pseudocyst & Percutaneously drained. \\
\hline Patient 4 & 6000 & Epigastric pain & 206 & 18.35 & $\begin{array}{l}\text { Interstitial oedematous } \\
\text { pancreatitis }\end{array}$ & Conservative treatment \\
\hline Patient 5 & 6000 & Upper abdominal pain & 248 & 20.07 & $\begin{array}{l}\text { Interstitial oedematous } \\
\text { pancreatitis }\end{array}$ & Conservative treatment \\
\hline Patient 6 & 6000 & Upper abdominal pain & 233 & 33 & $\begin{array}{l}\text { Interstitial oedematous } \\
\text { pancreatitis }\end{array}$ & Conservative treatment \\
\hline
\end{tabular}

receive a full course on asparaginase [17]. As a clinically significant rise in serum amylase is usually only seen after 7 days of initial presentation of abdominal pain, serial serum amylase alone is inadequate to confirm the diagnosis. We suggest CRP level as additional tool for diagnosis and monitoring of improvement. Imaging in the form of CT abdomen is more useful to assist diagnosis rather than depending on mere ultrasound in our series. CT abdomen should also be performed to assess severity or sequelae of pancreatitis when clinical presentation worsened. In our series, triad of positive symptoms and signs, elevated CRP and positive sign of pancreatitis on CT abdomen have brought improvement in diagnosing this difficult pathology. Serial level of CRP on downward trend also assisted in affirming improvement in patients' clinical conditions.

\section{References}

1. Quintanilla-Flores DL, Flores-Caballero MA, Rodriguez-Gutierrez R, Tamez-Pérez HE, González-González JG (2014) Acute Pancreatitis and Diabetic Ketoacidosis following L-Asparaginase/Prednisone Therapy in Acute Lymphoblastic Leukemia. Case Rep Oncol Med Article ID 139169. [Crossref]

2. Flores-Calderón J, Exiga-Gonzaléz E, Morán-Villota S, Martín-Trejo J, YamamotoNagano A (2009) Acute Pancreatitis in Children With Acute Lymphoblastic Leukemia Treated With L-Asparaginase. J Pediatr Hematol Oncol 31: 790-793. [Crossref]

3. Clavell LA, Gelber RD, Cohen HJ, Hitchcock-Bryan S, Cassady JR, et al. (1986) Fouragent induction and intensive asparaginase therapy for treatment of childhood acute lymphoblastic leukemia. $N$ Engl J Med 315: 657-663. [Crossref]

4. Haskell CM, Canellos GP, Leventhal BG, Carbone PP, Block JB, et al. (1969) $\mathrm{L}$-asparaginase: therapeutic and toxic effects in patients with neoplastic disease. $N$ Engl J Med 281: 1028-1034. [Crossref]

5. Jaffe N, Traggis D, Das L, Moloney WC, Hann HW, et al. (1971) L-asparaginase in the treatment of neoplastic diseases in children. Cancer Res 31: 942-949. [Crossref]

6. Nesbit M, Chard R, Evans A, Karon M, Hammond GD (1979) Evaluation of intramuscular versus intravenous administration of L-asparaginase in childhood leukemia. Am J Hematol Oncol 1: 9-13. [Crossref]

7. Alvarez OA, Zimmerman G (2000) Pegaspargase-induced pancreatitis. Med Pediatr Oncol 34: 200-205. [Crossref]

8. Weetman RM, Baehner RL (1974) Latent onset of clinical pancreatitis in children receiving L-asparaginase therapy. Cancer 34: 780-785. [Crossref]

9. Knoderer HM, Robarge J, Flockhart DA (2007) Predicting asparaginase-associated pancreatitis. Pediatr Blood Cancer 49: 634-639. [Crossref]

10. Susan L. Kearney, Suzanne E. Dahlberg, Donna E. Levy (2009) Clinical Course and Outcome in Children with Acute Lymphoblastic Leukemia and AsparaginaseAssociated Pancreatitis. Pediatr Blood Cancer 53: 162-167.

11. Morimoto T, Hirai K, Fukumura A, Takakura H, Koike T, et al. (2013) Early diagnosis of asparaginase-associated pancreatitis based on elevated serum elastase-1 levels: Case reports. Biomed Rep 1: 651-653. [Crossref]

12. Abhayakumar SM, Purohit S, Arunakumari BS, Lakshmaiah KC, Appaji L (2013) L-asparginase induced pseudopancreatic cyst: a rare case report. Indian J Surg Oncol 4 313-315. [Crossref]

13. Barreto SG, Rodrigues J (2007) Comparison of APACHE II and Imrie Scoring Systems in predicting the severity of Acute Pancreatitis. World J Emerg Surg 2: 33. [Crossref]

14. Blamey SL, Imrie CW, O'Neill J, Gilmour WH, Carter DC (1984) Prognostic factors in acute pancreatitis. Gut 25: 1340-1346. [Crossref]

15. Fisic E, Poropat G, Bilic-Zulle L, Licul V, Milic S, Stimac D (2013) The Role of IL-6, 8, and 10, sTNFr, CRP, and Pancreatic Elastase in the Prediction of Systemic Complications in Patients with Acute Pancreatitis. Gastroenterol Res Pract Volume 2013.

16. Oh HJ, Im SA, Lee JW, Chung NG, Cho B (2014) Relationship between modified CT severity index and clinical features of L-asparaginase-associated pancreatitis in pediatric acute lymphoblastic leukemia. Pediatr Hematol Oncol31: 647-655. [Crossref]

17. Silverman LB, Gelber RD, Dalton VK, Asselin BL, Barr RD, et al. (2001) Improved outcome for children with acute lymphoblastic leukemia: results of Dana-Farber Consortium Protocol 91-01. Blood 97: 1211-1218. [Crossref]

Copyright: (C2016 Aziz DA. This is an open-access article distributed under the terms of the Creative Commons Attribution License, which permits unrestricted use, distribution, and reproduction in any medium, provided the original author and source are credited. 